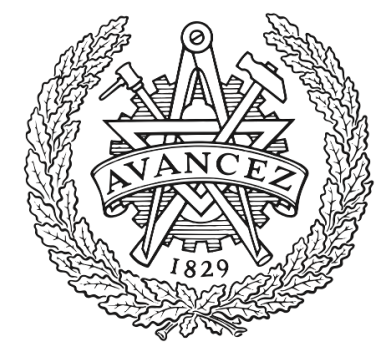

CHALMERS

UNIVERSITY OF TECHNOLOGY

\title{
High-order photoelectron holography in the midinfrared-wavelength regime
}

Downloaded from: https://research.chalmers.se, 2023-04-26 07:49 UTC

Citation for the original published paper (version of record):

Agueny, H., Hansen, J. (2018). High-order photoelectron holography in the midinfrared-wavelength regime. Physical Review A, 98(2). http://dx.doi.org/10.1103/PhysRevA.98.023414

N.B. When citing this work, cite the original published paper. 


\title{
High-order photoelectron holography in the midinfrared-wavelength regime
}

\author{
Hicham Agueny ${ }^{1, *}$ and Jan Petter Hansen ${ }^{2}$ \\ ${ }^{1}$ Department of Physics and Technology, Allegaten 55, University of Bergen, N-5007 Bergen, Norway \\ ${ }^{2}$ Department for Space, Earth and Environment Science, Chalmers University of technology SE-412 96 Gothenburg, Sweden
}

(Received 23 February 2018; revised manuscript received 28 May 2018; published 16 August 2018)

\begin{abstract}
We study the dynamical photoelectron holography of an excited hydrogen atom with a strong midinfrared laser field using numerical solutions of the three-dimensional time-dependent Schrödinguer equation. A clear holographic interference pattern of first and high order (hologram) is recorded in the two-dimensional (2D) momentum distribution of the photoelectron. The patterns are well reproduced by additional quantitative calculations based on the Lippmann-Schwinger equation. Here, the high-order interference effect is linked to the multsicattering of low-momentum electrons driven by the midinfrared laser field prior to ejection. The phenomenon manifests by low-momentum structures in 2D momentum distributions and is found to be sensitive to the change of the optical carrier-envelope phase (CEP). By analyzing the temporal evolution of the buildup of the hologram, we show that this sensitivity results from the birth time of the continuum wave packet with an offset in time during the subcycle dynamics, thus encoding information about the ionization mechanism which is mapped into the CEP-resolved 2D momentum distributions and angle-resolved photoelectron spectra. It is indeed an indicator that the ejected electrons exhibit a memory of their birth-time. These findings suggest that the CEP-resolved photoelectron holography serves as a tool for a direct measurement of attosecond dynamics. Furthermore, the effects due to such multiple scattering electron paths can be used to design new atom interferometers to highlight mechanisms that require higher accuracy.
\end{abstract}

DOI: 10.1103/PhysRevA.98.023414

\section{INTRODUCTION}

Advances in ultrafast laser technology have opened up the possibility of seeing the dynamical behavior of electrons in atoms [1], molecules [2], and condensed matter [3]. Comprehensive knowledge of this dynamics provides an unprecedented insight into the quantum structure and physical properties of quantum systems under study, enabling to coherently control their dynamical behavior. Recent developments of innovative techniques based on ultrafast electron diffraction have enabled direct access to the electron dynamics with subatomic resolution in both space and time [4-6]. Electron holography is one such technique [7]. It is based on the formation of an interference pattern or "hologram" enabling to record the image of the medium used. Recently, strong-field photoelectron holography has been used to probe the ultrafast dynamics of atoms [8]. A hologram was recorded which provides insight into the structural information of the parent ion with spatiotemporal resolution [8] and allows to learn about the laser-driven electron rescattering process itself [9].

Following these pioneering works, many studies have been reported for different systems and various regimes of strongfield ionization, allowing to further understand the complete nature of the mechanism underlying the obtained holographic structures [10-15]. In all these studies, the main focus was to learn about the scattering event as the electron scatters once by the parent ion. Recently, it has been shown that electron holography in strong-field ionization of an aligned $\mathrm{N}_{2}$ molecule

\footnotetext{
*hicham.agueny@uib.no
}

provides information about the continuum wave packet [16]. Here the released electron wave packet exhibits an offset in the phase front, which modifies the rescattering process. Molecular alignment dependence of the photoelectron holography found to be originated from the ionization mechanism rather than the scattering event [16].

Motivated by the general interest of photoelectron holography to provide high spatiotemporal resolutions in diffraction as well as in imaging and spectroscopy, we address in the present work the high-order photoelectron holography which offers a detailed view of the electron dynamics. The high-order effects here are related to the multsicattering of electrons driven by the laser field prior to ejection, and manifest by low-momentum dynamical structures. The underlying dynamics offers the possibility to learn about the continuum wave packet, which can be monitored by varying the properties of the laser fields.

In general, the outgoing wave packet generated via strongfield ionization is presumed to instantly follow the temporal evolution of the laser field. This suggests that the so-called optical carrier-envelope phase (CEP), which determines the timing of the field oscillations with respect to the pulse peak [17], can be used to tackle the generated wave packet. Here, by changing the optical phase, electrons are born with an offset in time and get ionized with a time delay that occurs during the subcycle dynamics. This feature, indeed, should be mapped out in the measured photoelectron momentum distribution and identified clearly in the angle-resolved photoelectron holography.

In this work, a ten-cycle midinfrared laser pulse is used to ionize an excited state of the hydrogen atom having a low ionization potential $(1.51 \mathrm{eV})$. This combination leads 
to a large spatial excursion of the electron and has been considered a suitable way to record holographic fringes with higher visibility $[8,11]$. By solving the three-dimentional (3D) time-dependent Schrödinguer equation (TDSE), we show that a change in the optical phase affects the holographic patterns of first and high order observed in the two-dimensional (2D) momentum distribution. We further show that these features can be reflected in the angle-resolved photoelectron spectrum. The sensitivity of these distributions to the CEP is found to be a signature of a change in the timing of the birth of the continuum wave packet, which can be exploited to learn about the ionization mechanism.

The paper is organized as follows. In Sec. II, we provide the theoretical basis for the electron dynamics, which is based on the 3D-TDSE and Lippmann-Schiwnger equation. Section III is devoted to the analysis of the two-dimensional momentum distributions of the photoelectron, and particularly to the temporal evolution of the buildup of the hologram during the subcycle dynamics and its sensitivity to the optical phase. Finally, conclusions are given in Sec. IV. Atomic units are used in this article unless otherwise indicated.

\section{THEORETICAL MODEL AND IMPLEMENTATION}

\section{A. Time-dependent Schrödinguer equation calculations}

The TDSE for a hydrogen atom interacting with a laser field is expressed in cylindrical coordinates and is written as

$$
\left[H_{0}+H_{I}(t)-i \frac{\partial}{\partial t}\right] \Psi(\vec{r}, t)=0 .
$$

where $\vec{r}=(\rho, z, \phi)$ denotes the vector position of the electron, and is defined in cylindrical coordinates. $H_{0}$ is the field-free Hamiltonian transformed into

$$
H_{0}=-\frac{1}{2} \frac{\partial^{2}}{\partial z^{2}}+-\frac{1}{2} \frac{\partial^{2}}{\partial \rho^{2}}+\frac{\left(m^{2}-\frac{1}{4}\right)}{2 \rho^{2}}-\frac{1}{\sqrt{\rho^{2}+z^{2}}},
$$

by scaling the wave function in Eq. (1) according to $\Psi(\vec{r})=$ $\psi(\rho, z) e^{i m \phi} / \sqrt{2 \pi \rho}$. Here, $m$ stands for the magnetic quantum number and only $\mathrm{m}=0$ is considered through our investigation. This is because of the cylindrical symmetry of the system due to the fact that the laser field in the time-dependent interaction $H_{I}(t)$ is assumed to be linearly polarized along the $z$ axis. This interaction is treated in the velocity gauge and can be expressed within the dipole interaction as

$$
H_{I}(t)=-i \vec{\nabla} \times \vec{e}_{z} \frac{E_{0}}{\omega} g(t) \cos \left(\omega t+\phi_{\mathrm{CEP}}\right),
$$

where $E_{0}$ is the maximum field strength and $\omega$ is the angular

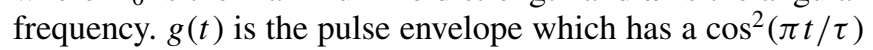
form with a duration of $\tau=2 \pi N c / \omega$. Here, Nc $f$ is the total number of cycles. $\phi_{\mathrm{CEP}}$ is the CEP of the laser pulse, and determines the offset between the pulse peak and the nearest maximum of the oscillating electric field. It provides information about the temporal evolution of the field.

In our simulation, we consider a 10-cycle midinfrared light pulse at a central wavelength of $3.5 \mu \mathrm{m}$ (corresponding to a photon energy of $0.354 \mathrm{eV}$ with a single cycle time of $11.7 \mathrm{fs}$ ) and a peak intensity of $3.510^{12} \mathrm{~W} / \mathrm{cm}^{2}$. Under these conditions, and according to he Keldysh parameter $\gamma=\sqrt{I_{p} / 2 U_{p}} \approx$
0.44 , we define the ionization process to be in the tunneling regime. Here, $I_{p}=1.51 \mathrm{eV}$ is the ionization potential and $U_{p}=E_{0}^{2} / 4 \omega \approx 4 \mathrm{eV}$ is the electron ponderomotive energy in a laser field.

We consider the $3 d$ states to be the initial states. The time evolution of the electronic wave function $\psi(\rho, z)$, which satisfies the TDSE [cf. Eq. (1)], is solved numerically using a split-operator method based on the fast Fourier transform algorithm [18]. This is carried out on a grid of size $|z|=4096$ and $|\rho|=2048$ a.u. with the spacing grid $\delta z=\delta \rho=0.25$ a.u., i.e., 16384 and 8192 grid points along the $z$ - and $\rho$-axis direction, respectively.

We stress here that the centrifugal term in Eq. (2), which is attractive for $m=0$, is singular at $\rho=0$. To avoid this numerical problem, we double the grid over to the negative $\rho$ side, and considere the antisymmetrical wave packets with respect to the $\rho$ coordinate since the symmetrical ones do not satisfy the boundary condition at $\rho=0$ [19].

The time step used in the simulation is $\delta t=0.04$ a.u. The convergence is checked by performing additional calculations with twice the size of the box and a smaller time step $(\delta t / 2)$. A detailed study of the numerical convergence is provided in the Supplemental Material [20]. An absorbing boundary is employed to avoid artificial reflections, but without perturbing the inner part of the wave function. The boundary is chosen to span $10 \%$ of the grid size in each direction. At the end of the interaction $t=t_{f}$, we calculate the two-dimensional momentum distribution of the photoelectron from the Fourier transform of the spatial ionization wave function. This last is obtained by projecting the time-dependent wave packet onto the continuum of the atomic system. This is done by extracting the important bound states, which are obtained using imaginary time propagation.

\section{B. Lippmann-Schwinger equation calculations}

We give a brief description of the Lippmann-Schwinger equation (LSE) used for a two-body system to support our TDSE calculations. Assuming that the scattering system is considered unaffected by the laser field, one can use the time-independent LSE and the total electron wave function can be written as

$$
|\psi\rangle=|\phi\rangle+\frac{1}{E-H_{0}+i \varepsilon} V|\psi\rangle,
$$

where we take the limit $\varepsilon \longrightarrow 0^{+}$. Here $H_{0}$ is the free-particle Hamiltonian, $V$ is the scattering potential, and $E$ is the energy of the system $H_{0}+V$. The above equation takes the following form in the position representation:

$$
\psi(\vec{r})=\phi(\vec{r})+\int d \vec{r} G\left(\vec{r}-\vec{r}^{\prime}\right) V\left(\vec{r}^{\prime}\right) \psi\left(\vec{r}^{\prime}\right),
$$

where $G(\vec{r})=-e^{i p_{i}|\vec{r}|} / 2 \pi|\vec{r}|$ is the Green's function (freepropagator operator). For an initial plane wave with momentum $\vec{p}_{i}$, Eq. (5) becomes

$$
\psi(\vec{r})=\frac{1}{(2 \pi)^{3 / 2}} e^{i \vec{p}_{i} \vec{r}}+f\left(\vec{p}_{f}\right) \frac{e^{i p_{i} r}}{r},
$$




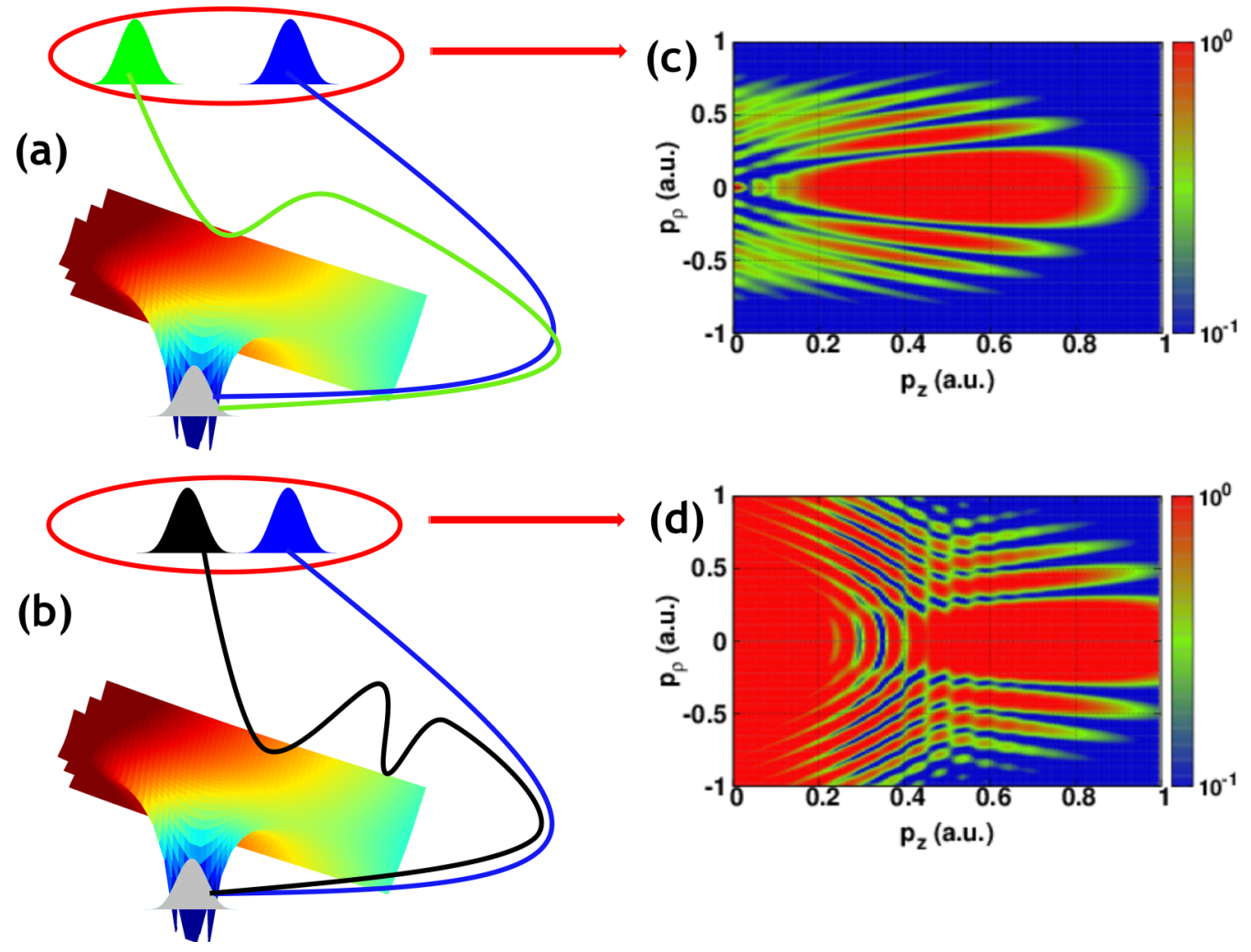

FIG. 1. (a) Schematic diagrams showing single scattering and (b) multiple scattering paths of the electron prior to emission from a symmetric atomic potential. Here the (blue) curve illustrates the unscattered electron path, while the (green) curve and (black) curve illustrate single scattering and multiple scattering electron paths, respectively. Calculations of the electron density in momentum space stemming from the Lippmann-Schwinger equation Eq. (9) for (c) single scattering and (d) where multiple scatterings are included. See text for details and discussion about the model.

where $f\left(\vec{p}_{f}\right)$ is the scattering amplitude

$$
f\left(\vec{p}_{f}\right)=-(2 \pi)^{-1} \int d \vec{r}^{\prime} e^{-i \vec{p}_{f} \vec{r}^{\prime}} V\left(\vec{r}^{\prime}\right) \psi\left(\vec{r}^{\prime}\right) .
$$

The first term in Eq. (6) is related to the unscattered plane wave, while the second term refers to the scattered spherical wave with momentum $\vec{p}_{f}$, where its magnitude is $p_{f}=p_{i}$ for the elastic scattering.

The wave function in Eq. (6) can be generalized for the unperturbed incident electron wave packet by considering a coherent superposition of the wave function in Eq. (6). The fully coherent wave function can be written in the following form:

$$
\psi(\vec{r}, t)=\int d \vec{k} a(\vec{k}) e^{i \vec{k}^{2} t / 2}\left[\frac{1}{(2 \pi)^{3 / 2}} e^{i \vec{k} \vec{r}}+f\left(\vec{k}_{f}\right) \frac{e^{i k r}}{r}\right],
$$

where $a(\vec{k})$ is the momentum amplitude of the incident electron and is assumed to have a Gaussian form $a(\vec{k})=$ $\left(2 \pi \sigma^{2}\right)^{-1 / 2} e^{-\left(\vec{k}-\vec{p}_{i}\right)^{2} \sigma^{2} / 2}$. Here $\sigma$ determines the width of the wave packet and $p_{i}$ is its central momentum. Due to the localization of the Gaussian wave packet around $\vec{k} \simeq \vec{p}_{i}$, Eq. (8) can be simplified

$$
\begin{aligned}
\psi(\vec{r}, t)= & \frac{1}{\left(2 \pi \sigma^{2}\right)^{1 / 2}} e^{i \vec{p}_{i}^{2} t / 2}\left[\frac{1}{(2 \pi)^{3 / 2}} e^{i \vec{p}_{i} \vec{r}} e^{-\left(\vec{r}-\vec{r}_{i}\right)^{2} / 2 \sigma^{2}}\right. \\
& \left.+f\left(\vec{p}_{f}\right) \frac{e^{i p_{i} r}}{r} e^{-\left(r-r_{i}\right)^{2} / 2 \sigma^{2}}\right],
\end{aligned}
$$

where we expand the phase $g(\vec{k})=i\left(k r+\vec{k}^{2} t / 2\right)$ in Eq. (8) about $\vec{p}_{i}$ using the expansion

$$
g(\vec{k}) \simeq g\left(\vec{p}_{i}\right)+\left(\vec{k}-\vec{p}_{i}\right) \cdot \vec{\nabla}_{\vec{k}} g(\vec{k})+O\left(\vec{k}-\vec{p}_{i}\right)^{2},
$$

and $\vec{r}_{i}=\vec{p}_{i} t$ is the position of the scatter.

The total wave function in Eq. (9) is a coherent superposition of unscattered and rescattered wave packets, and their interference should exhibit fringe patterns in analogy with electron holography [7]. The wave function contains information about the electron-potential rescattering and reveals the important features of the multiple scattering effects via the scattering amplitude $f\left(\vec{p}_{f}\right)$. The restriction to the first-order approximation on the amplitude $f\left(\vec{p}_{f}\right)$ reveals signatures only about the single scattering. This model helps to identify the multiple scattering effects, which should be present in the 2D photoelectron momentum distribution obtained from our TDSE calculations.

Equation (9) is solved by iteration, and the convergence is reached after four iterations. Here the Gaussian parameters are chosen to mimic the laser-driven electron rescattering conditions [16]. The initial position of the wave packet is set to be $\left(\rho_{i}=0, z_{i}=-58\right.$ a.u. $)$, which corresponds to the maximum spatial electron excursion $\alpha=E_{0} / \omega^{2}$. Its momentum $p_{i}$ is related to the classical $2 U_{p}$ cutoff energy $\left(p_{i}=\sqrt{2 U_{p}}=\right.$ 0.54 a.u.). The width of the wave packet is fixed at $\sigma=4.3$ a.u.. Here we assume a longitudinal distribution for the initial 
electron wave packet, while the returning electron is supposed to have a transversal distribution.

\section{RESULTS AND DISCUSSION}

Photoelectron holography has been discussed widely both experimentally and theoretically, and its origin is understood as a consequence of the interference between scattered and unscattered electrons during the subcycle dynamics $[8,9]$. The interference results in a holographic pattern which can be observed in the 2D momentum distributions. The induced phenomenon has been observed at various wavelengths and intensities of the laser field (i.e., different regimes of strongfield ionization). The basic concept of the phenomenon in the tunneling regime is illustrated in Fig. 1(a). Here, two main contributions lead to the emergence of the holographic pattern. The first contribution comes from the electron path that leads to a direct ionization via the tunneling effect (blue curve); the second one is due to the electron wave packet which after gets tunnel-ionized it scatters by the parent ion once (green color) before it joins the continuum with the same final momentum as the first electron path. Because of the phase accumulated between these two paths during the subcycle dynamics they will interfere, giving rise to a hologram analogous to the one in the original idea of electron holography [7]. The resultant phenomenon refers to us the photoelectron holography of first order since the electron scatters once by the parent ion. The first observation of the phenomenon was reported by Huismans and coworkers [8] by measuring the photoelectron momentum distributions for xenon atoms ionized with intense $7 \mu \mathrm{m}$ midIR lasers. The observation was confirmed through a theoretical model based on a phase difference between two different paths followed by the electron to end up with same final momentum, and where one of the paths involves rescattering by the parent ion [8].

In the same way, photoelectron holography of higher order can occur. The phenomenon is linked here to the multiple scattering of electrons driven by the laser field prior to ejection. The schematic diagram of the effect is depicted in Fig. 1(b) and shows the electron path (black curve) undergoing multiple scattering before it ends in the continuum. Multiscattering effects were found to manifest by a low-energy structure in the experimental photoelectron angular distributions generated by mid-IR laser from xenon and argon [9]. The effect was confirmed by a simple model of strong-field ionization based on a superposition of plane and spherical photoelectron waves. It has been shown that this feature is a signature that an electron can pass by its parent ion more than once before strongly scattering from it [9].

To provide deeper insight into these higher-order phenomena and disentangle multiple scattering from single scattering effects, we perform calculations based on the LSE [cf. Eq. (9)]. When solving the LSE within the first-order approximation, only the single scattering effect is taken into account. Here we assume the longitudinal distribution for the initial electron wave packet, which moves in the $z$ direction and scatters in an arbitrary direction. The resulting electron density in momentum space is displayed in Fig. 1(c) and shows clear lobeside fringes similar to those observed in [8]. By iterating
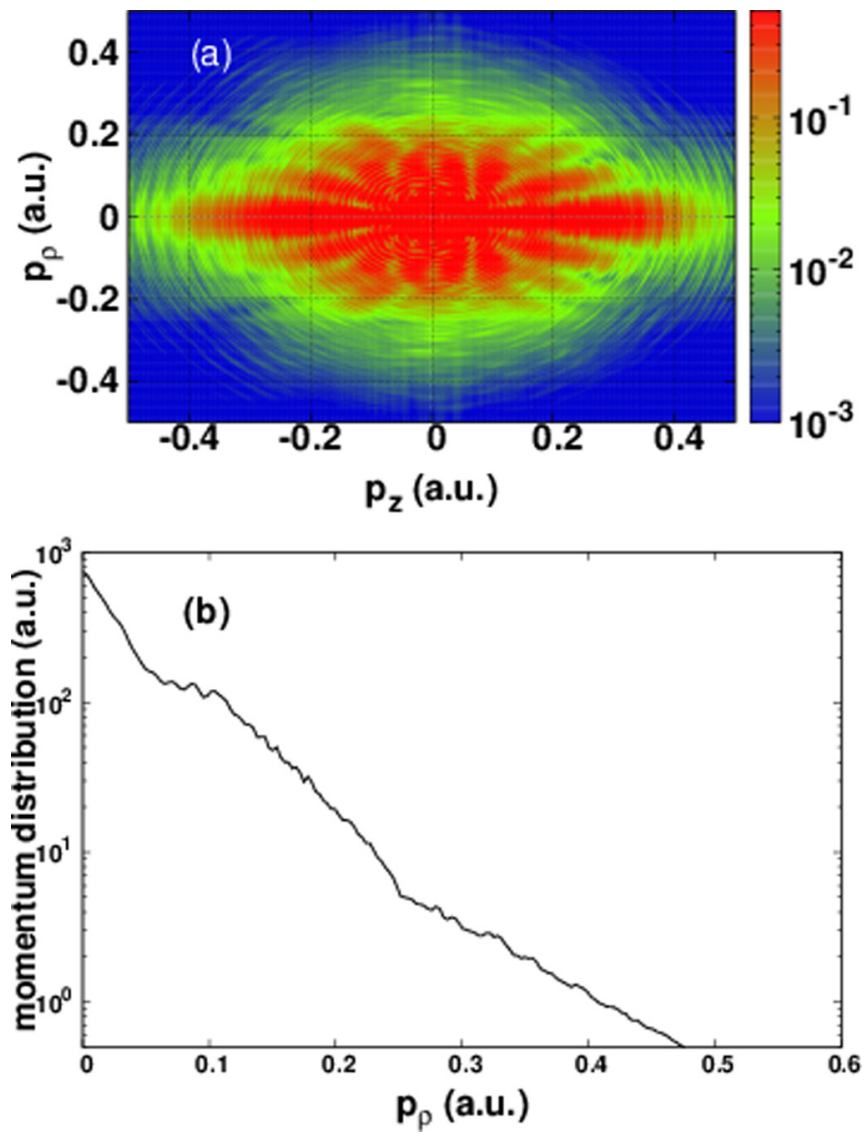

FIG. 2. (a) CEP-integrated 2D-photoelectron momentum distribution on the $p_{z}-p_{\rho}$ plane for the initial $3 d$ state of the hydrogen atom. (b) 1D-momentum distribution as a function of the transversal momentum $p_{\rho}$ for a fixed longitudinal momentum at $p_{z}=0.1$ a.u.

the LSE beyond the first-order approximation and up to the fourth-order, the multiple scattering of the electrons are explicitly included. Here, the returning electron is supposed to have a transversal distribution. The electron density stemming from these calculation is plotted in Fig. 1(d) and shows additional structures which emerge at the low-momentum region. The model, therefore, captures the general features of a hologram and will help to identify theme in the 2D photoelectron momentum distributions.

We first study the CEP-integrated momentum distribution obtained when an excited hydrogen atom is illuminated by a midinfrared light pulse. The TDSE calculations for this distribution are performed for the initial $3 d$ state and are shown in Fig. 2(a). We use a laser wavelength of $3500 \mathrm{~nm}$ (photon energy $0.354 \mathrm{eV}$ with a single cycle time of $11.7 \mathrm{fs}$ ) and a peak intensity of $3.510^{12} \mathrm{~W} / \mathrm{cm}^{2}$. The calculations are performed with ten optical cycles and the distribution in Fig. 2 is averaged over different values of CEP covering the range $[0, \pi]$. As seen in Fig. 2(a), the distribution shows rich structures manifested by lobeside fringes, which are similar to those observed experimentally in $[8,9]$ and are attributed to the holographic interference patterns.

As in [9] we distinguish two different types of the patterns: interference stripes at the outer momentum region $\left(\left|p_{z}\right|>\right.$ 0.15 a.u.), which are well reproduced by the model based 

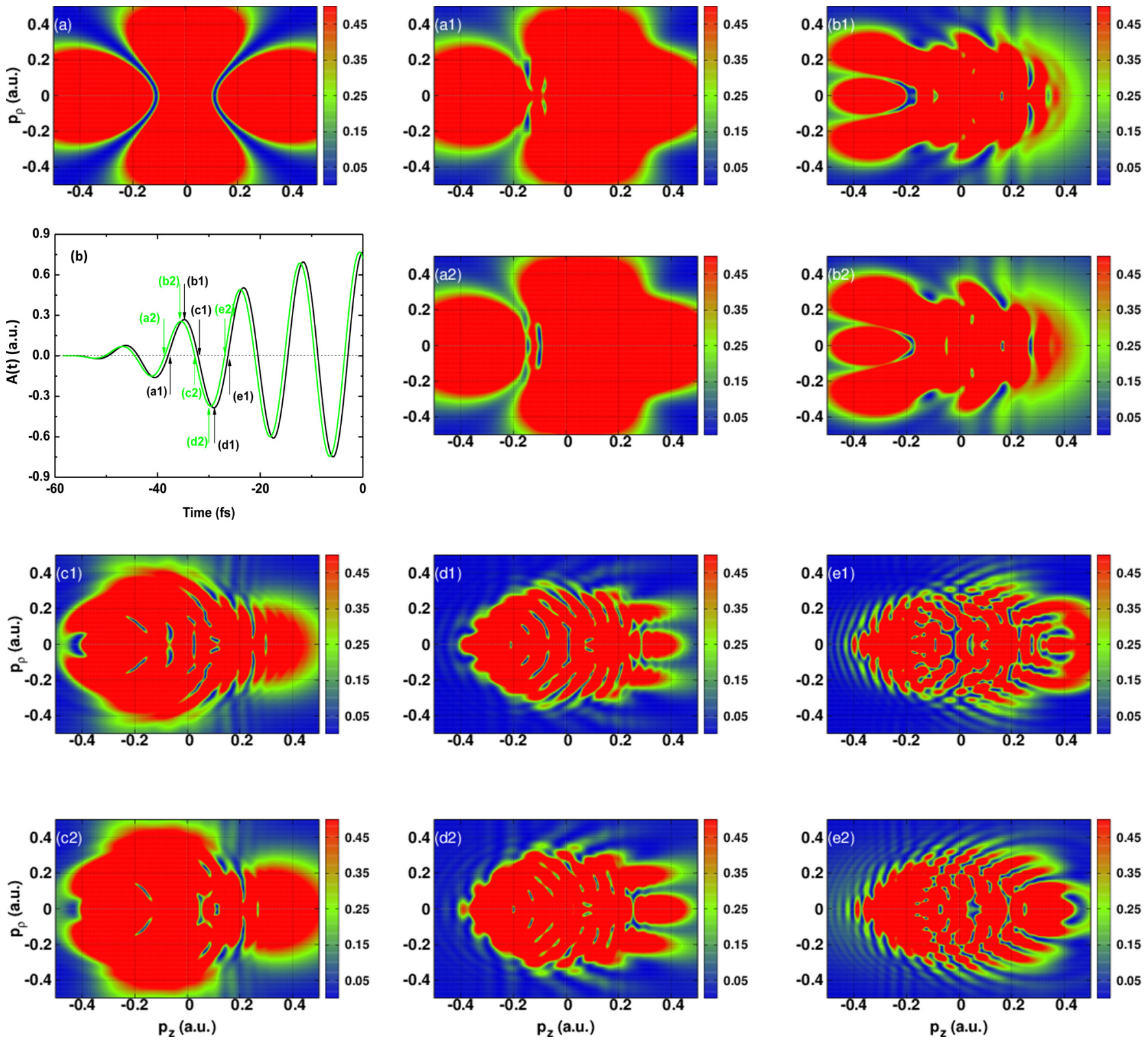

FIG. 3. Snapshots of the electron-probability density on the $p_{z}-p_{\rho}$ plane. (a): initial orbital $3 d$. [(a1)-(e1)]: $\phi_{\text {CEP }}=0$; [(a2)-(e2)]: $\phi_{\mathrm{CEP}}=0.11 \pi(642.6 \mathrm{as})$. (b) Vector potential with cosine-shaped waveforms (here only the first half of the total period of the pulse is plotted). Arrows indicate different times of the electron ionization that correspond to the snapshots $[(\mathrm{a} 1)-(\mathrm{e} 1)]$ and $[(\mathrm{a} 2)-(\mathrm{e} 2)]$. $\phi_{\mathrm{CEP}}=0$ (black solid line); $\phi_{\mathrm{CEP}}=0.11 \pi$ (green solid line).

on the LSE for the single scattering process [cf. Fig. 1(c)] and additional fringe patterns emerge at the inner momentum region $\left(\left|p_{z}\right|<0.15\right.$ a.u.), which are distributed along the momentum transverse direction. The inner-region pattern is well reproduced by the LSE for multiple scatterings as shown in Fig. 1(d), and therefore should be linked to the multiscattering of electrons by the parent ion prior to the ejection, as illustrated in Fig. 1(b). Similar effects have been discussed in connection with ion-impact induced ionization [21,22], which were observed at low-momenta of the ejected electron.

The low-energy structure in Fig. 2 manifests by modulations in the one-dimensional (1D) momentum distribution when it is plotted as a function of the transverse momentum $p_{\rho}$ for a fixed longitudinal momentum $p_{z}$ (here $p_{z}=0.1$ a.u.). The modulations emerge in the transverse momentum region $p_{\rho}<$ 0.2 a.u. and are shown in Fig. 2.

In the following, we investigate in more detail the CEPresolved photoelectron holography of first and high order, and focus on four optical phases where the rescattering time covers a range of few attoseconds (as) to few femtoseconds (fs). In general, the produced patterns carrier information not only about the scattering object but also about the ionization mechanism. Insight into these events can be achieved by examining the CEP dependence of the electron dynamics. Indeed, the change on the optical phase leads to a time-varying ionization of the electron. This is because the continuum wave packet born at the time $t$ will born with an offset at the time $t+\tau$, where $\tau=\phi_{\mathrm{CEP}} / \omega$. Therefore, electrons will be generated with 

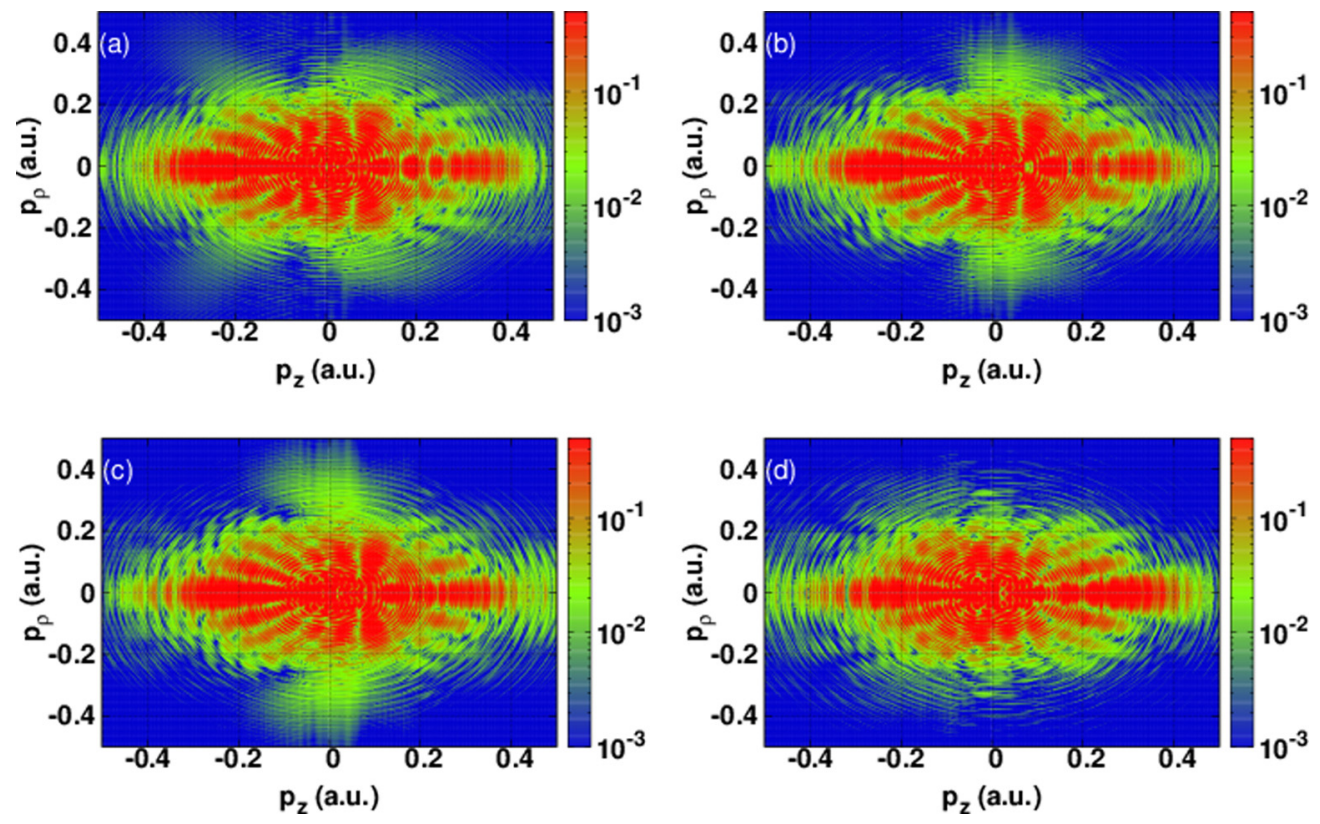

FIG. 4. Photoelectron momentum distribution showing the holographic interference at different optical phases $\phi_{\mathrm{CEP}}$. (a) $\phi_{\mathrm{CEP}}=0$; (b) $\phi_{\mathrm{CEP}}=0.11 \pi(642.6 \mathrm{as})$; (c) $\phi_{\mathrm{CEP}}=0.25 \pi$ (1460.4 as); (d) $\phi_{\mathrm{CEP}}=0.5 \pi$ (2920.7 as).

different momenta depending on the optical phase. The change of the momentum of the electron can be described by the classical equation of motion $\Delta \vec{p}\left(t_{\text {ionz }}\right)=\int_{t_{\text {ionz }}}^{\infty} E(t) d t$, where $t_{\text {ionz }}$ is the ionization time. The final electron's momentum $\vec{p}_{f}\left(t_{\text {ionz }}\right)$ is then given by

$$
\vec{p}_{f}\left(t_{\text {ionz }}\right)=\vec{p}_{i}+\Delta \vec{p}\left(t_{\text {ionz }}\right)=\vec{p}_{i}+A\left(\vec{t}_{\text {ionz }}\right),
$$

where $\vec{p}_{i}$ is the initial momentum. It becomes clear that the change on the final momentum is directly related to the vector potential, and hence preserves the temporal information of the electric field. Accordingly, by manipulating the optical phase one can follow the temporal evolution of the field and hence get insight into the ionization mechanism. In the following, we will show that the footprint of this mechanism is mapped in the $2 \mathrm{D}$ momentum distributions and can be resolved through the change of the optical phase.

The dynamical access to these processes is provided by looking at the temporal evolution of the electron wave packet. This is shown in Figs. 3(a1) to 3(e1) and Figs. 3(a2) to 3(e2) in momentum space, respectively, for the two optical phases 0 and $0.11 \pi$ (the corresponding rescattering time is 642.6 as). The figures represent snapshots of the buildup of the hologram during one optical cycle, as depicted by arrows in Fig. 3(b).

Figure 3(a) shows the electron density before the dynamics starts out. The probability densities in Figs. 3(a1) and 3(a2) and Figs. 3(b1) and 3(b2) capture the propagation of the electron wave packet at the first quarter-cycle of the optical cycle at zero and at the maximum of the vector potential, respectively [cf. Fig. 3(b)]. It is clearly seen that at the maximum vector potential the electron density is drastically changed during the quarter cycle with the emergence of fringe patterns that occur mainly in the forward direction. The pattern is found to be slightly dependent on the optical phase. During the second half-cycle of the pulse [cf. Figs. 3(c1) to 3(e2)] the electron density substantially changes with the optical phase. This change reflects the attosecond evolution of the continuum wave packet which is born with an offset in time (here the offset is 642.6 as). As time evolves, the electron probability density shows rich structures manifested by sidelobe fringes. Here, two different types of fringes emerge: those localized at the inner momentum region $\left(0<p_{z}<0.2\right.$ a.u. $)$ and those at the outer region $\left(0.2<p_{z}<0.4\right.$ a.u. $)$. The emergence of these fringes reflects the origin of the observed patterns in Fig. 2 which is linked to the interference between scattered (or rescattered) and unscattered electron paths, and shows

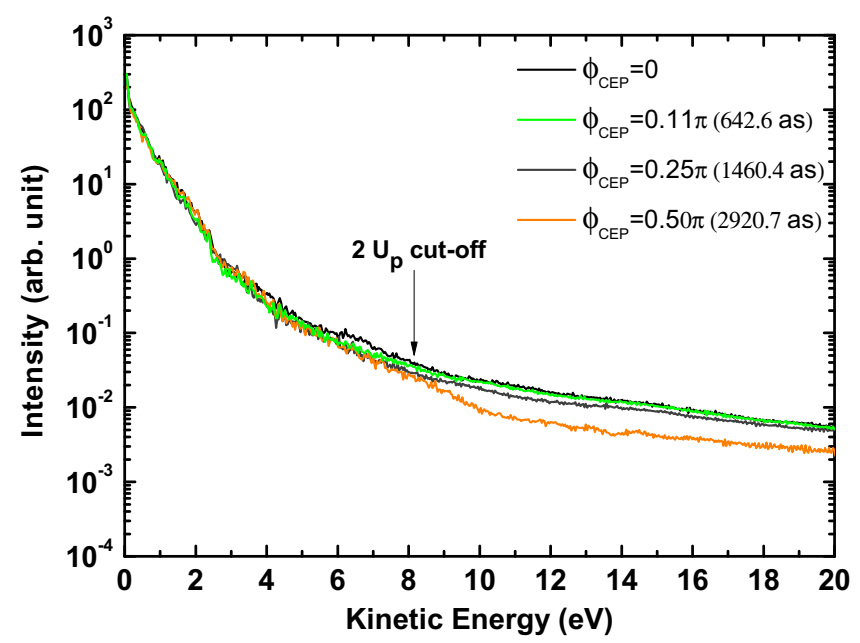

FIG. 5. Angle-averaged photoelectron spectra at different optical phases $\phi_{\mathrm{CEP}}$, and their corresponding times (timing of the field oscillations with respect to the pulse peak) also are indicated between parentheses. The arrow indicates the energy corresponding to the classical $2 U_{p}$ cutoff energy. 

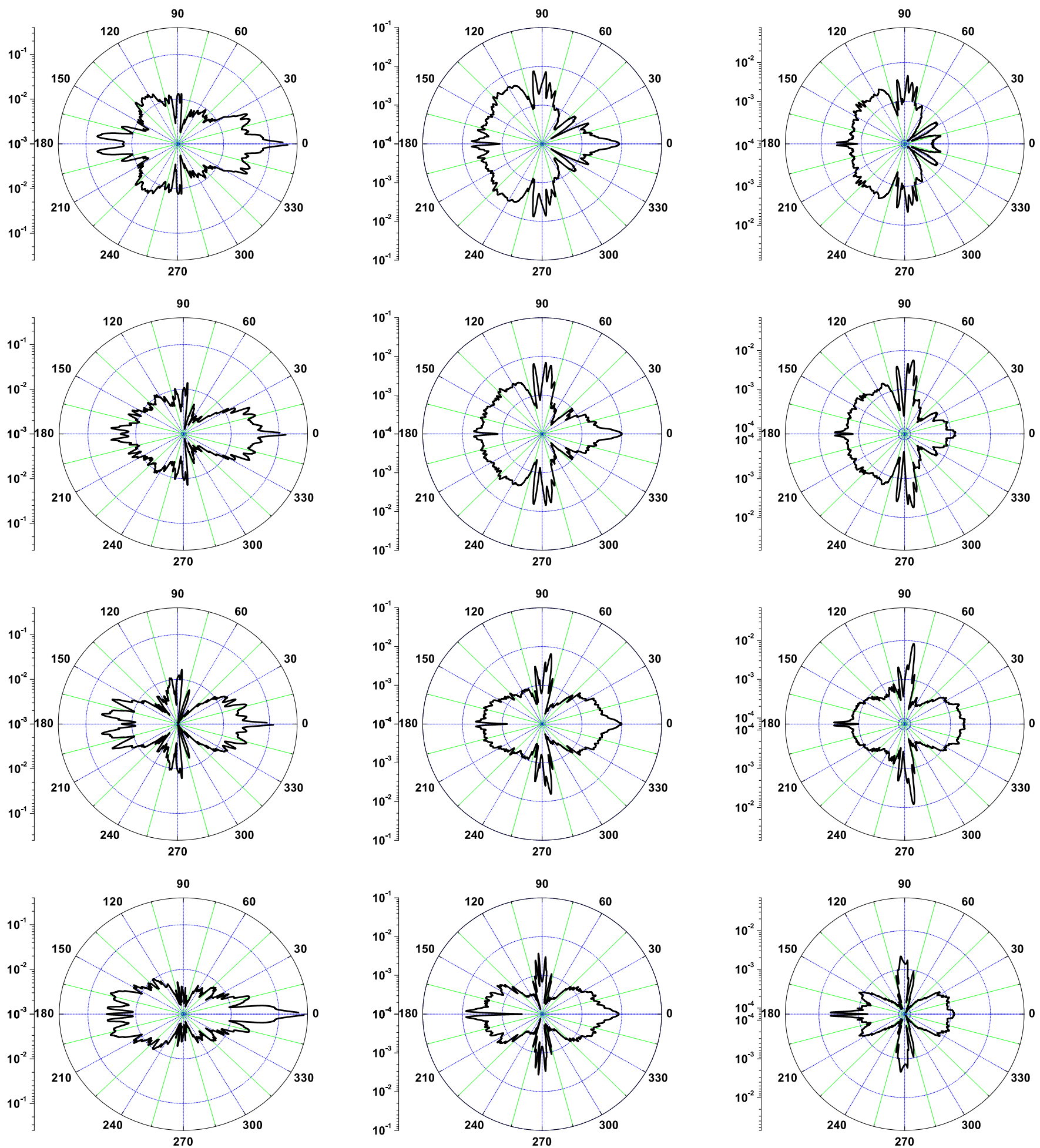

FIG. 6. Angle-resolved photoelectron holography spectra at different optical phases $\phi_{\mathrm{CEP}}$ and various photoelectron energies. Rows from the top to the bottom correspond to the optical phases: $0,0.11 \pi$ (642.6 as), $0.25 \pi$ (1460.4 as), and $0.5 \pi$ ( 2920.7 as), respectively. Columns from the left-hand side to the right-hand side correspond to the photoelectron energies: 4,8 , and $10 \mathrm{eV}$.

that the interference phenomenon occurs during the subcycle dynamics. The structures are well reproduced by calculations based on LSE where multiple scattering are included as shown in Fig. 1(d). Once again, the shape of the fringes and fringe spacing are found to be sensitive to the optical phase. Indeed, owing to the nature of the oscillating electric field, electrons are generated during the subcycle dynamics with different momenta causing multiple scattering events. As a consequence, various levels of holographic pattern are built up in the time domain, preserving thus the footprint of the birth-time of the continuum wave packet that is sensitive to the change of the optical phase. This change modifies, in turn, 
the ionization mechanism, and should be mapped into the momentum distributions. Thus, this results suggests that the attosecond evolution of the continuum wave packet can be clearly identified in the CEP-resolved momentum distributions. These distributions are shown in Fig. 4 for different optical phases 0, $0.11 \pi$ (642.6 as), $0.25 \pi$ (1460.4 as), and $0.5 \pi$ (2920.7 as). Here, the attosecond signal is provided by various changes in the lobeside structures and fringe spacing which are signatures of subcycle dynamics interference of first and higher order. Therefore, by manipulating the optical phase one can precisely tailor the optical cycles to yield to an unprecedented degree of control for the subcycle dynamics interference. Furthermore, interferences between electrons which are born with an offset in time yield insight into the ionization event. This is reflected in the optical phase dependence of the holographic patterns, which offers an opportunity for the measurement of subcycle duration [23].

At this point we conclude that the changes in the shape of the fringes in the hologram by varying the optical phase are signatures of a phase shift of the birth-time of the continuum wave packet during the subcycle dynamics. This sensitivity is an important feature of the ejected electrons preserving information about the ionization mechanism. It is indeed a signature that the ejected electron exhibits a memory of its birth-time. Furthermore, the dependence of the optical phase upon the ultrashort time electron dynamics is a timing indicator of the ultrafast electron emission.

For completeness, we emphasize in the remaining part of this section the angle-integrated and angle-resolved photoelectron holography spectra. The optical phase dependence upon the angle-integrated photoelectron spectra is displayed in Fig. 5, and shows rapid decay of the ionization rate with the kinetic energy, as one can expect at long wavelength regime. By varying the optical phase the angle-integrated spectra look identical at low kinetic energies below the classical cutoff energy, as indicated by arrow in Fig. 5, while the discrepancies emerge beyond this energy.

To provide more insight into this dependence, we consider angle-resolved photoelectron spectra for different photoelectron energies covering the low-energy region: 4,8 , and $10 \mathrm{eV}$ [cf. Fig. 6]. Although the effect of the change of the optical phase in the photoelectron spectra in Fig. 5 is somewhat small at the low-energy region, the angular distribution shows a strong sensitivity to the optical phase. It can be seen that the angle-resolved photoelectron spectra consist of rich structures in the forward and backward directions of the emitted photoelectron with the emergence of a substantial asymmetric profile, reflecting the observed hologram in the 2D momentum distribution. Furthermore, additional features are observed in the transverse direction and found to be influenced by the change of the optical phase. These structures reflect the inner-region patterns observed in the 2D momentum distributions [cf. Figs. 2 and 4], which are distributed along the momentum transverse, and are signatures of multiscattering effects. These observations enable us to track the released wave packet via the angle-resolved photoelectron holography by varying the optical phase. On the other hand, the changes in the distributions are much larger in comparison to the angle-integrated spectra [cf. Fig. 5]. This suggests that the angle-resolved photoelectron spectrum is more powerful to study the electron dynamics than the angle-integrated ones.

\section{CONCLUSION}

In conclusion, we investigate the optical phase dependence upon the dynamical photoelectron holography of high order (hologram) using a midinfrared laser pulse. On the basis of the solutions of the 3D-TDSE for the excited $3 d$ state of the hydrogen atom, the 2D momentum distribution is carried out and found to record a clear photoelectron holographic fringes. We are able to identify two types of fringes: those localized in the inner-momentum region which result from multiscattering of electrons driven by infrared laser field, and those in the outer-region that correspond to a single scattering process. These findings are validated by additional calculations based on the Lippmann-Schwinger equation. We further show that these multiscattering effects are mapped out in the angular distribution in the transverse direction of the photoelectron emission. The time-dependent buildup of the hologram was studied and has been shown to provide insight into the ionization event through the change of the optical phase. This last is found to modify the holographic fringes owing to an offset in time of the birth of the continuum wave packet during the subcycle dynamics. This sensitivity is, indeed, a signature that the ejected electron exhibits a memory of its birth-time. Thus, by exploring the sensitivity of the photoelectron holography to the optical phase, the footprint of the continuum wave packet, which is encoded in the hologram, can be manipulated offering thus an opportunity for the measurement of subcycle duration. Furthermore, the effects due to such multiple scattering phenomena may lead to new applications of atom interferometers allowing to make evidence of mechanisms that require higher accuracy.

\section{ACKNOWLEDGMENTS}

The research has been supported by UNINETT Sigma2 AS which manages the national infrastructure for computational science in Norway. Numerical calculations were carried out at the Cray XE6 (Hexagon) supercomputer installation at Parallab at the University of Bergen (UiB).
[1] E. Goulielmakis, Z.-H. Loh, A. Wirth, R. Santra, N. Rohringer, V. S. Yakovlev, S. Zherebtsov, T. Pfeifer, A. M. Azzeer, M. F. Kling, S. R. Leone, and F. Krausz, Real-time observation of valence electron motion, Nature 466, 739 (2010).

[2] C. I. Blaga, J. Xu, A. D. DiChiara, E. Sistrunk, K. Zhang, P. Agostini, T. A. Miller, Louis F. DiMauro, and C. D. Lin, Imaging ultrafast molecular dynamics with laser-induced electron diffraction, Nature 483, 194 (2012).

[3] A. L. Cavalieri, N. Mller, Th. Uphues, V. S. Yakovlev, A. B. Caronka, B. Horvath, B. Schmidt, L. Blümel, R. Holzwarth, S. Hendel, M. Drescher, U. Kleineberg, P. M. Echenique, R. Kienberger, F. Krausz, and U. Heinzmann, 
Attosecond spectroscopy in condensed matter, Nature 449, 1029 (2007).

[4] A. H. Zewail, Four-Dimensional Electron Microscopy, Science 328, 187 (2010).

[5] F. O. Kirchner, A. Gliserin, F. Krausz, and P. Baum, Laser streaking of free electrons at $25 \mathrm{keV}$, Nat. Photon. 8, 52 (2014).

[6] S. Haessler, J. Caillat, W. Boutu, C. Giovanetti-Teixeira, T. Ruchon, T. Auguste, Z. Diveki, P. Breger, A. Maquet, B. Carré, R. Taïeb, and P. Salières, Attosecond imaging of molecular electronic wavepackets, Nat. Phys. 6, 200 (2010).

[7] A. D. Gabor, A new microscopic principle, Nature 161, 777 (1948).

[8] Y. Huismans, A. Rouzée, A. Gijsbertsen, J. H. Jungmann, A. S. Smolkowska, P. S. W. M. Logman, F. Lépine, C. Cauchy, S. Zamith, T. Marchenko, J. M. Bakker, G. Berden, B. Redlich, A. F. G. van der Meer, H. G. Muller, W. Vermin, K. J. Schafer, M. Spanner, M. Yu. Ivanov, O. Smirnova, D. Bauer, S. V. Popruzhenko, and M. J. J. Vrakking, Time-Resolved Holography with Photoelectrons, Science 331, 61 (2011).

[9] D. D. Hickstein, P. Ranitovic, S. Witte, X.-M. Tong, Y. Huismans, P. Arpin, X. Zhou, K. Ellen Keister, C. W. Hogle, B. Zhang, C. Ding, P. Johnsson, N. Toshima, M. J. J. Vrakking, M. M. Murnane, and H. C. Kapteyn, Direct Visualization of Laser-Driven Electron Multiple Scattering and Tunneling Distance in Strong-Field Ionization, Phys. Rev. Lett. 109, 073004 (2012).

[10] X.-B. Bian, Y. Huismans, O. Smirnova, K.-J. Yuan, M. J. J. Vrakking, and A. D. Bandrauk, Subcycle interference dynamics of time-resolved photoelectron holography with midinfrared laser pulses, Phys. Rev. A 84, 043420 (2011).

[11] T. Marchenko, Y. Huismans, K. J. Schafer, and M. J. J. Vrakking, Criteria for the observation of strong-field photoelectron holography, Phys. Rev. A 84, 053427 (2011).

[12] X.-B. Bian and A. D. Bandrauk, Attosecond Time-Resolved Imaging of Molecular Structure by Photoelectron Holography, Phys. Rev. Lett. 108, 263003 (2012).

[13] Y. Huismans, A. Gijsbertsen, A. S. Smolkowska, J. H. Jungmann, A. Rouzée, P. S. W. M. Logman, F. Lépine, C. Cauchy, S. Zamith,
T. Marchenko, J. M. Bakker, G. Berden, B. Redlich, A. F. G. van der Meer, M. Yu. Ivanov, T.-M. Yan, D. Bauer, O. Smirnova, and M. J. J. Vrakking, Scaling Laws for Photoelectron Holography in the Midinfrared Wavelength Regime, Phys. Rev. Lett. 109, 013002 (2012).

[14] Y. Li, Y. Zhou, M. He, M. Li, and P. Lu, Identifying backwardrescattering photoelectron hologram with orthogonal two-color laser fields, Opt. Express 24, 23697 (2016).

[15] S. G. Walt, N. Bhargava Ram, M. Atala, N. I. ShvetsovShilovski, A. von Conta, D. Baykusheva, M. Lein, and H. Jakob Wörner, Dynamics of valence-shell electrons and nuclei probed by strong-field holography and rescattering, Nat. Commun. 8, 15651 (2017).

[16] M. Meckel, A. Staudte, S. Patchkovskii, D. M. Villeneuve, P. B. Corkum, R. Dörner, and M. Spanner, Signatures of the continuum electron phase in molecular strong-field photoelectron holography, Nat. Phys. 10, 594 (2014).

[17] F. Krausz and M. Ivanov, Attosecond physics, Rev. Mod. Phys. 81, 163 (2009).

[18] M. Feit, J. Fleck, Jr., and A. Steiger, Solution of the Schrödinger equation by a spectral method, J. Comput. Phys. 47, 412 (1982).

[19] E. Fattal, R. Baer, and R. Kosloff, Phase space approach for optimizing grid representations: The mapped Fourier method, Phys. Rev. E 53, 1217 (1996).

[20] See Supplemental Material at http://link.aps.org/supplemental/ 10.1103/PhysRevA.98.023414 for details of the numerical convergence.

[21] H. Agueny, A. Makhoute, A. Dubois, and J. P. Hansen, Coherent electron emission beyond Young-type interference from diatomic molecules, Phys. Rev. A 93, 012713 (2016).

[22] H. Agueny and J. P. Hansen, Quantum multiscattering interferences in collision-induced coherent electron emission from diatomic molecules by swift ion impact, Phys. Rev. A 94, 052702 (2016).

[23] J. Itatani, F. Quéré, G. L. Yudin, M. Yu. Ivanov, F. Krausz, and P. B. Corkum, Attosecond Streak Camera, Phys. Rev. Lett. 88, 173903 (2002). 